THEORIA ET HISTORIA SCIENTIARUM, VOL. VI, N²

Ed. Nicolas Copernicus University 2002

Koichiro Matsuno

\title{
Beyond Representation: Bridging the Chasm between the Different Grammatical Tenses
}

\begin{abstract}
Representing the activity of experiencing in the empirical world is a formidable task because it requires a representation that remains passively immobile on its own. The activity of experiencing is about the concrete particulars addressed only in the present progressive and in the present perfect tense. The representation will come to be conceivable if the concrete particulars addressed in the present perfect happen to be identical to those addressed also in the present progressive tense. The equality serves as a universal in the present tense. Unless the present perfect tense is equated to the present progressive in the present tense, there would be no representation. Nonetheless, there is a possibility to think of a representation under the condition that it can frequently be updated. Constant update of a representation proceeds in the present progressive tense carrying the capacity of ameliorating those inconsistencies among the completed local events registered in the preceding present perfect tense. Short-lived representation can be appreciated only when we understand and view the present tense from the internalist perspective. The internalist present tense actualizes only in the limited interval over which the present perfect tense locally happens to be identical to the present progressive. Representation in the internalist present tense can cope with intrinsically dynamic situations through undermining the present tense while constantly eliminating the conflicts between the underlying present progressive and present perfect tense.
\end{abstract}

\section{Introduction}

When the issue of evolutionary processes is focused, it has been taken as a standard practice to represent them in observations and statements made in the present tense. This linguistic stipulation however comes to furnish evolutionary 
processes to be addressed with an artifact exclusively of linguistic origin (Matsuno, 1995b, 1998b; Van de Vijver et al, 1998). Take, for instance, an endeavor of representing evolutionary processes in terms of both natural selection and random mutation. Furthermore, just for the sake of argument for the time being, let us accept the following two statements made in the present tense. One is that natural selection is the process of selective retention of heritable traits, and the other is that random mutation is the process of random substitution of base pairs on DNA molecules (Matsuno, 1984). Although these universal statements made in the present tense seem legitimate in themselves, they are neither evolutionary nor historical of themselves in their concrete contents (Salthe, 1993). The two statements of a universal character must be supplemented by other empirical observations on the concrete particulars in order to demonstrate their real significance, if any.

At this point, we notice that the general universal in the present tense is supplemented by the concrete particulars on completed empirical observations registered in the present perfect tense. This representation of evolutionary processes in terms of the general universal in the present tense supplemented by the concrete particulars in the present perfect tense however raises a serious question of its legitimacy (Emmeche \& Hoffmeyer, 1991). It cannot address, as a matter of principle, the question of whether the general universal could remain invulnerable to the concrete particulars that could enrich their content as with the progression of evolutionary processes. If the general universal is of a hypothetical nature, the extent of its applicability to real empirical observations would extremely be limited. On the other hand, if the statement of a universal character is an inductive consequence of the preceding vast empirical observations, the likelihood of its alternation by others could not be eliminated. Any statement made in the present tense, if theoretically conceived, makes itself atemporal or ahistorical though it remains universal. At the same time, any empirical statement made in the present tense as an empirical induction cannot be universal enough though it remains legitimate to the extent of the accepted induction.

Representing evolutionary processes in the present tense thus begs the question of how and to what extent the statements in the present tense employed for this endeavor could be universal (Gendlin, 1995; Rosen, 1997). The role of a statement in the present tense for representing evolutionary processes is quite subtle. If it is too universal, the statement cannot be empirical enough to face the reality. Likewise, if it is too empirical, the statement cannot serve as a universal vehicle for representing evolutionary processes in the present tense.

We in this article wish to ameliorate the malaise surrounding the issue of representing evolutionary processes. The standard scheme of supplementing statements made in the present tense by empirical observations registered in the present perfect tense, though quite widely prevailing, still suffers from the 
ambivalent character of the present tense (Matsuno, 1999a). Our objective is to try to reach evolutionary processes as closely as possible without recourse to the scheme employing the present tense supplemented by the present perfect tense.

\section{Experiencing, Transforming and Representing}

Evolutionary process in the making is uncompleted movement in progress that can be addressed in the present progressive tense. What is unique to the present progressive tense is enhancement of the extent of concretization with the progression. Concretization proceeds with measurement internal to any participating material bodies in the sense that measurement comes to constrain the concerned parties more and more with its progression by distinguishing before and after the very act of each measurement (Pattee, 1977). This form of measurement internal to any material bodies, or internal measurement in short, is in fact ubiquitous wherever interactions of material origin are available (Matsuno, 1985, 1989; Rossler, 1987). Internal measurement is thus about the process of any material body being constrained by any other in whatever way. One party comes to identify another to the extent of being constrained by the latter (Matsuno, 1996a, 1998c).

What is more, measurement as a material movement distinguishes between measurement in progress and measurement in the product. This distinction will become crucial when representation of a measurement is focused. In view of the fact that representation is consistent in itself and remains passively immobile on its own, what can be represented is a measurement in the product instead of that in progress.

Measurement in progress that is referred to in the present progressive mode, on the other hand, inevitably lacks its global consistency. It does not fulfill the condition for serving as a representation of something that remains in itself globally consistent. There is no material means of coordinating everything in progress globally on the spot. Concurrent activities in the present progressive mode are necessarily conflicting with each other because of the absence of any material means for the global coordination (Gunji, 1995; Taborsky, 1997). However, insofar as there remains a globally consistent record of completed events registered in the present perfect mode, what is being in conflict and inconsistent in the concurrent activities in progress is constantly being moved in the progressive mode and being passed forward without being left behind in the record. There is no room of inconsistencies in stasis, otherwise there would be no record to rely upon.

The factor driving the present progressive mode forward is accordingly seen in the leftover of inconsistencies that cannot be frozen in the global record of 
completed events (Matsuno \& Salthe, 1995). The non-frozen leftover driving the subsequent progressive movement is in a form of constantly migrating inconsistencies. Any local act for a consistency turns out to be a cause for disturbing the preceding consistency in the neighborhood (Matsuno, 1996b, 1997a). This migration process of local inconsistencies is the rule of measurement in progress. Migrating inconsistencies are both the cause and the effect of moving the present progressive mode. In short, suppose I am walking through the crowd as avoiding collisions with others. I recognize that others are doing the same. This means that everybody walking in the crowd is constantly being part of the moving obstacle in the form of migrating inconsistencies towards everybody else there. Accordingly, measurement in progress cannot properly be represented as much as inconsistencies cannot be represented consistently. Focusing upon measurement in the product as a representation requires seeing how measurement in progress comes to be related to that in the product.

In particular, any material body interacting with others comes to experience inconsistencies in their progressive mode. If it can survive at least for some time, such a material body could serve as a representation of what it comes to have accomplished so far (Hoffmeyer, 1996). This observation implies that the material body experiencing inconsistencies originating in the neighborhood has the capacity of transforming itself into a consistent body. The transformed body in turn serves as a representation to be experienced by any material bodies in the neighborhood (Matsuno, 2000).

Measurement in progress on an arbitrary material body starts from experiencing representations of material bodies presented from the neighborhood in the form of inconsistencies. It is then followed by transforming itself into a consistent body, which is again to be presented to the others in the neighborhood as a representation. More specifically, what is available as the measurement in the product serving as a representation is the material body having transformed itself into a consistent one, however, only for a very short while before its inevitable next updating. Although measurement in progress faces migrating inconsistencies everywhere, they would disappear only temporarily during the limited period over which each material body can transform itself into a consistent one after experiencing inconsistencies with others.

One can thus note that the triad of experiencing, transforming and representing upholds measurement underlying mutually interacting bodies. Experiencing others in the form of migrating inconsistencies distinguishes between the present progressive and the present perfect tense because there is no equality in their implications between the two grammatical tenses in the presence of the inconsistencies. The present perfect tense does not carry agential capacity to modify what it has already implied any further. Inequality between the present progressive and the present perfect tense also applies to transforming since there 
is a distinct contrast between what has been completed and what has not yet been completed. Despite these differences, representation in the triad of experiencing, transforming and representing remains internally immobile on the part of a material body that has just transformed itself into a consistent body, at least until its inevitable next updating. Representation grounded upon the temporal equality between the present progressive and the present perfect tense can now be addressed in the present tense only internally. That is the internalist present tense.

The internalist present tense anchored at a representation in the triad of experiencing, transforming and representing is however dynamic in itself as carrying migrating inconsistencies both as the cause for and as the consequence of updating the internalist representation. This exhibits a marked contrast to the standard externalist present tense, in which the descriptive subject is supposed to be able to descriptively represent the object available out there in the present tense. No inconsistency is allowed in the externalist present tense. In addition, the externalist present tense assumes globally synchronous time applicable to the whole descriptive object because the acclaimed present tense is shared by all of the members constituting the entire object. This contrast between the internalist and the externalist present tense comes to furnish measurement in progress with a unique dynamic characteristic.

\section{Intensive versus Extensive}

Measurement in progress is concrete enough in the sense that a material body measuring another body is constrained by the latter. In fact, any concrete movement in progress is intensive in its concretization while it can be extensive in the product at least in the probabilistic or statistical sense if an ensemble of concretizations is available. The occurrence of extensive variables, however, should not be underestimated simply because they are merely derivatives from intensities. Extensive variables can furnish measurement in the product with its quantification. The contrast between extensive variables and intensities acting among themselves now raises a serious question of how both could develop in time in the dynamics giving measurement in progress grounded upon the triad of experiencing, transforming and representing.

Extensive variables are intrinsically local because the additive units are actualized only in the spatio-temporal dimensions locally. The internalist representation of a material body both measuring and being measured by others can be accomplished by referring to the associated local extensive variables in the present perfect tense. However, the internalist representation makes its constant updating inevitable because those material bodies in the neighborhood experience 
it in the form of migrating inconsistencies. We thus note that inconsistencies among the local extensive variables registered in the local present perfect mode drive the dynamics of intensities from within so as to leave none of them behind in the completed global record.

Intensities in the triad of experiencing, transforming and representing are intrinsically global in the sense that they cannot be cut into pieces, otherwise the associated migrating inconsistencies would forcibly be locally isolated in each piece. Nonetheless, what these inconsistencies are all about remains indefinite because the migrating inconsistencies are necessarily beyond its descriptive perspicuity. Intensities associated with the migrating inconsistencies are thus universally unique and singular. In other words, the dynamics of intensities is about a singular universal. This exhibits a distinct contrast to general universals with the dynamics of extensive variables, in the latter of which these variables are taken to be derivatives from the dynamics of intensities through an abstraction of dismissing the migrating inconsistencies.

Consider, for instance, Newton's laws of classical mechanics as a representative example of the general universal. The laws by themselves are not concrete enough to address the actual movement of material bodies in the empirical domain. The concretization requires the supplement of boundary conditions. Classical mechanics leaves out the process of concretization as maintaining that the process is not part of being dynamic. A similar remark also applies to quantum mechanics. The quantum mechanical equation of motion can concretely determine the wave function only when it is further adequately supplemented by its boundary conditions. The mechanistic abstraction of dynamics is peculiar in singling out part of the dynamics of intensities in the form of boundary conditions to independently be imposed upon the abstracted dynamics. This practice has worked as an extremely useful device to face a wide variety of material dynamics in the empirical world. Despite the success, however, the mechanistic abstraction does not serve as a guarantee dismissing the dynamics of intensities coping with the migrating inconsistencies as a singular universal.

The mechanistic abstraction makes the abstracted dynamics to be represented linguistically in a perspicuous manner as letting the law of motion be a formal cause while maintaining the boundary conditions to be an efficient cause (Rosen, 1991). The intensities ascribed to the boundary conditions are taken to be merely an efficient cause within the mechanistic abstraction. However, once the mechanistic stipulation is lifted, those intensities maintaining the migrating inconsistencies are no more efficient in their causation. They are material by themselves (Matsuno, 1997c). This implies that the dynamics of intensities is due to the material causation while the dynamics of extensive variables is taken to follow the formal cause supplemented by the efficient cause. The material thus carries with itself migrating inconsistencies. Any material body serves as 
a carrier of migrating inconsistencies. Rather, the dynamics of extensive variables comes to marginalize the covering dynamics of intensities into an efficient causation as embodied in the form of boundary conditions. The mechanistic abstraction unduly reduces material causation to efficient one. Unless the extensive stipulation is forcibly imposed, the dynamics remains intensive without prohibiting material causation from exercising its full capacity.

\section{Thermodynamics as an Intensive Dynamics}

A most significant attribute of the dynamics of intensities coping with the migrating inconsistencies is the material quantity called energy conceived within the framework of thermodynamics. The first law of thermodynamics addresses the transformation of the quality of energy, say, from mechanical to heat energy, while observing the conservation of its whole quantity in the completed record. Any material body comes to experience others in the neighborhood. The mediator is the migrating inconsistencies among themselves because of the absence of any material means for a global coordination on the spot. Then, it is going to transform itself into a consistent body to be presented to the others and to subsequently be experienced by the latter. The first law is about both experiencing and transforming in progress as referring to the representation in terms of energy that is constantly being updated (Matsuno, 1989). At the same time, the first law also refers to the conservation of energy in the completed record registered in the present perfect tense. What makes the first law of thermodynamics most peculiar compared to all of the other known laws of motion is in its unique juxtaposition of both the present progressive and the present perfect tense.

In contrast, mechanistic dynamics as exemplified in the form of the equation of motion, whether classical or quantal or whether non-relativistic or relativistic, is exemplified in the mode of the present tense. The mechanistic equation of motion has, however, originally been conceived as referring to the regularity underlying the completed record of events registered in the present perfect tense. One thus recognizes that mechanistic dynamics is anchored at a linguistic acrobatics of addressing the present perfect mode in the present tense. The linguistic acrobatics further forces us to admit that the future tense may also be referred to in the perfect mode, since any statement made in the present tense applies at any present moment. Even unwittingly, mechanistic dynamics lets the future already be perfected in the present within the record. Time in the mechanistic framework is no more than a subsidiary parameter detailing the perfected representation of the dynamics in the present. Once the mechanistic doctrine is adopted, the future eclipse of the sun has already been perfected and completed in the record available in the present. 
Thermodynamics certainly differs from mechanistic dynamics in admitting the dynamic movement that has not yet been perfected and completed. Uncompleted movement can be addressed in the present progressive mode in the form of migrating inconsistencies. This exhibits a marked contrast to completed movement in mechanistic dynamics. In the latter, any movement conceivable must necessarily be completed in the present tense because it addresses the movement transforming the already completed boundary conditions in the present tense. Compared to mechanistic dynamics carrying those events registered in the present perfect mode in the present tense, thermodynamics is distinctive in being carried by those migrating inconsistencies exclusively in the present progressive tense while precipitating the events frozen in the present perfect tense (Matsuno, 1998a, 1998d).

Thermodynamics upon uncompleted movement in progress is however related to mechanistic dynamics upon completed movement in the record in the sense that the events in the present progressive tense can necessarily be transferred into the record to the extent of having been completed so far. Uncompleted movement in progress does leave the completed part behind in the record while keeping itself constantly uncompleted. Occurrence of uncompleted movement persistently in progress in fact conforms to the linguistic practice of appreciating the present progressive mode that cannot completely reduce to the present perfect mode with its progression. In other words, the present progressive mode has to appreciate its conformity to the occurrence of the present perfect mode in retrospect. That means that the present progressive mode constantly drives itself so as to meet the constraints that the present perfect mode has to fulfill in any case. Since the conservation of energy in mechanistic dynamics is a stipulation met in the completed movement in the present perfect mode, the first law of thermodynamics is intensive or even intentional in its operation in fulfilling the conservation of energy a posteriori.

Intentionality is of course not limited to human consciousness. Intentionality requires those attributes of an agent, acts, an object to be acted upon, the noninferential criterion of intentionality, and the coherence criterion of sequences of these acts. Any agent carrying its own capacity of making choices is intentional in actualizing its consequence. Consider the sentence "The gazzele makes a quick turn in order to escape from the hunting panther." The gazelle maintains the noninferential criterion of making that particular quick turn in the sense that the turn it is making has not analytically been inferred from a necessary premise. It could turn otherwise. Furthermore, the gazelle can remain to be a surviving agent so long as it makes skillful turns frequently so as to fulfill the coherence criterion of escaping from the hunting panther.

The intentional attributes of an agent, acts, an object to be acted upon, the noninferrential criterion of intentionality and the coherence criterion of sequences 
of these intentional acts also apply to thermodynamics upon uncompleted movement in progress, especially in actualizing the first law of thermodynamics. The present progressive mode makes any participating material bodies agential because of the finiteness of the spatiotemporal horizon available to each of them. The agential activities manifest themselves in migrating inconsistencies. The noninferrential criterion of intentionality is found in fulfilling the conservation of energy in the record registered in the present perfect tense while the migrating inconsistencies are constantly being passed forward. Moreover, the present progressive mode drives itself forward while fulfilling the constraints that have to be met in the present perfect mode. Occurrence of the surviving present progressive mode in the face of the frozen present perfect mode manifests the coherence criterion of sequences of such intentional acts.

In essence, description in the present progressive tense is reductive in remaining reticent on and bracketing what the existence is all about, while the present progressive in itself is activity-oriented from the internalist perspective. A crucial issue at this point is how to refer to activities in progress in the present tense. Phenomenological reduction owing to Brentano and Husserl comes up with the notion of intentionality supplemented by a form of transcendental apriorism asking both the noninferential criterion of intentionality and the coherence criterion of sequences of intentional acts (Matsuno, 1999b). Compared to Husserlian transcendental phenomenology, the internalist stance is empirical in addressing activities in progress as referring to bridging the chasm between the present progressive and the present perfect tense in terms of migrating inconsistencies in the internalist present tense. Thermodynamics grounded upon uncompleted movement in progress admits bracketing what the whole existence is all about, but is intrinsically empirical and materialistic in grounding its intentionality upon bridging the different grammatical tenses, whose origin have been undoubtedly empirical to our human beings (Matsuno, 1995a, 1997b).

Thermodynamics processing the migrating inconsistencies is multi-agential since the dynamic inconsistencies manifest the occurrence of concurrent negotiation between more than one agents. Among them, the most significant agents are the supplier and the consumer of thermal energy (Matsuno and Swenson, 1999). There has been a theoretical tradition of making both the supplier and the consumer to be one and the same agent called a heat reservoir especially in the framework of equilibrium thermodynamics. But the strict association of the two cannot hold unless thermodynamic equilibrium is literally enforced in the actual situation. In fact, thermodynamics of the biosphere on the planet Earth is subject to the sun as the dominant energy supplier and to outer space as the ultimate energy consumer (Matsuno, 1996c). Equilibrium thermodynamics of course can be saved if both the supplier and the consumer are in complete equilibrium all through the time. However, once the disequilibrium between the 
two happens to occur by any chance, domination of one over the other could hold even temporarily. Thermodynamics admits in itself at least two possibilities of supplier- and consumer-domination. When it is out of equilibrium, thermodynamics thus comes to display irreversible characteristic either in the supplier- or the consumer-dominating manner (e.g., Imai et al, 1999).

Irreversible decay in the consumer-dominating thermodynamics proceeds as fast as possible. Lightning striking on the earth, that serves as the consumer of electrons, takes advantage of any chance available first. Thunderbolt never waits for the second chance to come. This observation is upon the remark that the migrating inconsistencies in the form of excess electric potential of the thundercloud drive themselves so as not to leave them behind in the completed record. In contrast, irreversible decay in the supplier-dominating thermodynamics proceeds as slow as possible. When the rate of sand fall from the above is fixed, the shape of the sand pile thus formed would be the one that remains least fragile. Irreversible decay in the supplier-dominating thermodynamics can be addressed by referring to the extensive quantities that have been supplied by whatever means. In contrast, irreversible decay in the consumer-dominating thermodynamics is intrinsically intensive in exhibiting the capacity of wanting for resources from within. Thermodynamics thus remains intensive unless the consumers are forcibly wiped out.

\section{Thermodynamic Intensities Tailoring Quantum Mechanics}

The first law of thermodynamics about experiencing and transforming energy while observing its quantitative conservation renders it possible to assimilate the transformed energy to a representation even temporarily until its inevitable subsequent updating is to come. In particular, the transformation of energy is imputed to the internally generated intensities processing the migrating inconsistencies. The internally generated intensities, when represented even temporarily, constitute the boundary conditions towards those thus entrained. Thermodynamics as an intensive dynamics accordingly comes to be an agency entailing boundary conditions. Actual implementation of such boundary conditions requires material hardware. At this point enters quantum mechanics (Matsuno and Paton, 1999). Since thermodynamic intensities manifest them in the consumer- dominating thermodynamics, thermodynamic upholding of boundary conditions necessitates quantum-mechanical implementation of consumers, or more specifically, energy consumers.

A most conspicuous example exhibiting energy consumption is exchange interaction of quantum-mechanical origin. Exchange interaction is capable of exchanging materials elements found in a material body with those to be found 
outside of the body. Any material consumer has the capacity of pulling in resourceful material elements from the outside and expelling waste elements to the outside. Exchange interaction just fulfills the role of implementing the agential capacity of consumption in quantum-mechanical terms.

An important aspect to note from the perspective of agency is that quantum mechanics by itself is undoubtedly mechanistic if it is subject to a fixed set of boundary conditions. There is no controversial argument against the mechanistic stipulation of quantum mechanics. Rather, what makes the material bodies implemented quantummechanically to be agential is thermodynamics as an intensive dynamics. The present emphasis on intensiveness in thermodynamics now urges us to see thermodynamics under a new light. The view letting thermodynamics be a derivative from quantum mechanics through statistical mechanics would turn out to be an inverted one. To the contrary, thermodynamics as an intensive dynamics utilizes quantum mechanics as a means of representing itself in material terms even though its constant updating becomes inevitable.

\section{Biology as a Natural Mediator between Thermodynamics and Quantum Mechanics}

Quantum mechanics can serve as a means of representing what thermodynamics is all about as being subject to its frequent updating. The underlying intensive capacity comes from thermodynamics as an intensive dynamics or more specifically from the consumerdominating thermodynamics. This is just one aspect of thermodynamics. One more aspect to be noted is seen in the supplier- dominating thermodynamics. Natural dynamics in the empirical domain is thus found in the interplay between the supplier- and the consumerdominating thermodynamics.

A most significant form of the consumer-dominating thermodynamics is found in Darwinian natural selection. It is natural selection which comes to influence over whichever organisms could survive as energy consumers in the long run. This is certainly a legitimate observation to be made within the realm of the consumer-dominating thermodynamics. Unless it is forcibly placed in the mechanistic context as is often the case with the neoDarwinian stipulation, natural selection could remain legitimate (Depew and Weber, 1995). Nonetheless, the consumer-dominating thermodynamics is only part of the whole argument. One more important player is the supplier-dominating thermodynamics.

A conspicuous example demonstrating the significance of the supplier- dominating thermodynamics in the realm of natural dynamics is found in ecology. Margalef (1968) observed that the irreversible decay rate of a unit biomass present in a pelagic ecosystem decreases with the progression of its 
succession. This observation manifests an empirical confirmation to that irreversible decay in the supplier-dominating thermodynamics proceeds as slow as possible.

We now encounter somewhat contradictory situation. Irreversible decay in the consumer-dominating thermodynamics proceeds as fast as possible, while that in the supplier-dominating one does as slow as possible (Ulanowicz, 1997). The present contradictory situation is however a reality. If the consumer domination lasts indefinitely without interruptions, the domination itself would eventually be terminated due to ultimate depletion of the resources to rely upon. Likewise, if the supplier-domination continues to survive for long, the ultimate steady state minimizing its irreversible decay rate depending exclusively upon how the resources are supplied would come to deny ever-changing evolutionary processes. If we view the evolutionary process taking place on our Earth as not yet being encountered with its ultimate stalemate, both the supplier- and the consumerdominating thermodynamics must have been alternated in a proper manner.

Thermodynamics as an intensive dynamics upheld upon the underlying migrating inconsistencies can be seen pretty random and incoherent if the scale of observation is limited both in space and in time (Nakajima, 1999). If only short-term inconsistencies are attended, it must be hard to envision the global organization that the thermodynamics could establish in the long run. These shortterm inconsistencies can be viewed as being supplied externally. In other words, the supplier-dominating thermodynamics could be relatively significant if the time scale of observation is limited. This remark is in conformity to the ecological observation saying the minimization of the irreversible decay rate of a unit biomass present in an ecosystem, since the time scale of ecological succession is quite small in its time scale compared to evolutionary time scale. However, once thermodynamic behaviors on the longer-time scale are focused, they may be influenced significantly by those of the consumers of material origin more significantly.

The inevitable interplay between the supplier- and the consumer-dominating thermodynamics can thus leave the imprinting of each uniqueness in the natural dynamics proceeding there. What is unique to the supplier-dominating thermodynamics is its stability or reliability because of the its capacity of mitigating the irreversible decay. In contrast, the consumer-dominating thermodynamics is unique in its plasticity and flexibility because of its intrinsic capacity of enhancing its irreversible decay. In reality, this pair of reliability and flexibility is in a tradeoff (Conrad, 1983). Both reliability and flexibility widely observed in the biological realm are in fact a representation of the underlying thermodynamics, and the material hardware has been recruited from quantum mechanics. 


\section{Concluding Remarks}

The descriptive scheme of representing an empirical phenomenon in the present tense supplemented by concrete observations made in the present perfect tense can remain diehard if the present tense is taken to address both the general universal and the concrete particulars. Mechanistic doctrine takes most advantage of this scheme. However, the priority of the present tense over the other grammatical tenses comes to beg the question of how the present tense dominates over the present progressive tense, for instance. The present progressive tense, in fact, serves as the most basic grammatical tense for addressing any activity of experiencing the empirical world since the experiencing is always about the concrete particulars. In fact, both the present progressive and the present perfect tense are about the concrete particulars because they are associated with concrete activities in the empirical domain. This observation in turn brings about a far- reaching consequence upon material dynamics.

If the equality between the present progressive and the present perfect tense in the present tense happens to hold by any chance, mechanistic scheme will naturally follow because it rests upon the linguistic stipulation of getting both the present progressive and the present perfect from the present tense. Quantum mechanics is in fact a supreme case of getting mechanistic dynamics from the equation of motion stated in the present tense. In contrast, thermodynamics is quite unique in its capacity of constantly ameliorating the conflicts between the present progressive and present perfect tense while updating the both. Homeostasis demonstrated in the form of sustaining fluctuations without failing to fulfill the first law of thermodynamics in the effect thus constitutes the boundary conditions towards the mechanistic dynamics proceeding there. Thermodynamics as a ceaseless activity for eliminating the conflicts between the present progressive and the present perfect tense provides quantum mechanics with its boundary conditions to face with. Biology is just a natural embodiment of demonstrating the interplay between thermodynamics and quantum mechanics.

Fundamental to the observation that thermodynamics constitutes the boundary conditions to the underlying quantum mechanics is the linguistic stipulation emphasizing the internalist perspective. The internalist raises the question of how in the world one can make statements in the present tense from within. This question is totally absent in the mind of the externalist who takes for granted the presence of the object to be stated at any present moment out there. Although both the present progressive and the present perfect tense are derivatives from the present tense in the mind of the externalist, the internalist takes a completely opposite perspective, namely, deriving the present tense from 
the interplay between the present progressive and the present perfect tense. It is this internalist perspective of linguistic origin which provides both thermodynamics and quantum mechanics with their respective proper roles of upholding biology in the actual empirical world.

\section{Referenences}

Conrad, M., 1983. Adaptability Plenum Press, New York.

Depew, D. J., and Weber, B, H., 1995. Darwinism Evolving: systems Dynamics and the Genealogy of Natural Selection (MIT Press, Cambridge Massachusetts).

Emmeche, C., and Hoffmeyer, J., 1991. From language to nature - the semiotic metaphor in biology. Semiotica 84, 1-42.

Gendlin, E. T., 1995. Crossing and dipping: some terms for approaching the interface between natural understanding and logical formulation. Minds and Machines 5, 547-560.

Gunji. Y.-P, 1995. Global logic resulting from disequilibration process. BioSystems 35, 33-62.

Hoffmeyer, J., 1996. Signs of Meaning in the Universe. Indiana University Press, Bloomington Indiana.

Imai, E., Honda, H., Hatori, K., Brack, A., and Matsuno, K., 1999. Elongation of oligopeptides in a simulated submarine hydrothermal system" Science 283, 831-833.

Margalef, R., 1968. Perspectives in Ecological Theory. University of Chicago Press, Chicago.

Matsuno, K., 1984. Open systems and the origin of proto-reproductive units. In: Beyond NeoDarwinism (Ho, M. W. \& Saunders, P. T. (Eds.)) (Academic Press, London), pp. 61-88.

Matsuno, K., 1985. How can quantum mechanics of material evolution be possible?: symmetry and symmetry-breaking in protobiological evolution. BioSystems $\backslash T, 179-192$.

Matsuno, K., 1989. Protobiology: Physical Basis of Biology, CRC Press, Boca Raton Florida.

Matsuno, K., 1995a. Consumer power as the major evolutionary force. J. Theor. Biol. 173, 137145.

Matsuno, K.., 1995b. Use of natural languages in modelling evolutionary processes. Proceedings of the $14^{\text {th }}$ International Congress of Cybernetics (International Association of Cybernetics, Namur Belgium), pp. 477-472.

Matsuno, K., 1996a. Internalist stance and the physics of information. BioSystems 38, 111-118.

Matsuno, K., 1996b. Symmetry in synchronous time and information in asynchronous time. Symmetry: Culture and Science 1, 295-305.

Matsuno, K., 1996c. Boltzmann's dynamics on the primitive earth about 3.8 billion years ago. In: Chemical Evolution: Physics of the Origin and Evolution of Life (Chela- Flores, J. \& Raulin, F. (Eds.)) (Kluwer, Dordrecht), pp. 231-238. 
Matsuno, K., 1997a. Information: Resurrection of the Cartesian physics. World Futures 49, 235-249.

Matsuno, K., 1997b. Biodynamics for the emergence of energy consumers. BioSystems 42, 119-127.

Matsuno, K., 1997c. Molecular semantics and the origin of life. BioSystems 42, 129-139.

Matsuno, K., 1998a. Dynamics of time and information in dynamic time. BioSytems 46, $57-71$.

Matsuno, K., 1998b. Competence of natural languages for describing the physical origin of life. In: Evolutionary Systems: Biological and Epistemological Perspectives on Selection and Self-Organization (Van de Vijver, G., Salthe, S. N. \& Delpos, M. (Eds.)) (Kluwer, Dordrecht), pp. 295-306

Matsuno, K., 1998c. Space-time framework of internal measurement. In: Computing Anticipatory Systems, AIP Conference Proceedings 437 (Dubois, D. M., (Ed.)) (American Institute of Physics, Woodbury New York), pp. 101-115.

Matsuno, K., 1998d. Emergent phenomena of time in quantum mechanics" Acta Polytechnica Sandinavica 91M, 61-70.

Matsuno, K., 1999a. The clock and its triadic relationship. Semiotica, 27, 433-452. Matsuno,

K., 1999b. Evolving life: constant turnover from inconsistencies to intensities. Analecta Husserliana, 901, 332-349.

Matsuno, K., 2000. The internalist stance: a linguistic practice enclosing dynamics. Ann. New York Acad. Sci., in press.

Matsuno, K., and Salthe, S. N., 1995. Global idealism/local materialism, Biol. Philos. 10, 309337.

Matsuno, K., and Paton, R. C., 1999. Quantum mechanics in the present progressive mode and its significance in biological information processing” BioSystems 49, 229-237.

Matsuno, K., and Swenson, R., 1999. Thermodynamics in the present progressive mode and its role in the context of the origin of life. BioSystems, 51, 53-61.

Nakajima, T., 1999. Biological probability: cognitive process of generating probabilites of events in biological systems. J. Theor. Biol. 200, 77-95.

Pattee, H. H., 1977. Dynamic and linguistic modes of complex systems. Int. J. Gen. Syst. 3, 259-266.

Rosen, R., 1991. Life Itself: a Comprehensive Inquiry Into the Nature, Origin and Fabrication of Life. Columbia University Press, New York.

Rosen, S. M., 1997. Wholeness as the body of paradox. J. Mind Behav. 18, 391-424. Róssler,

O. E., 1987. Endophysics, In: Real Brains, Artificial Minds (Casti, J. L. \& Karlqvist, A. (Eds.)) (North Holland, New York), pp. 25-46.

Salthe, S. N., 1993. Development and Evolution: Complexity and Changes in Biology. Bradford/The MIT Press, Cambridge Massachusetts.

Taborsky, E., 1997. The Textual Society. University of Toronto Press, Toronto. Ulanowicz,

R. E., 1997. Ecology, The Ascendent Perspective. Columbia University Press, New York.

Van de Vijver, G., Salthe, S. N., and Delpos, M. (Eds.), 1998. Evolutionary Systems: Biological and Epistemological Perspectives on Selection and Self-Organization. Kluwer, Dordrecht. 